\title{
STABILITY AND BIFURCATION OF ROTATING NONLINEARLY ELASTIC LOOPS
}

BY

\author{
TIMOTHY J. HEALEY
}

Cornell University, Ithaca, New York

1. Introduction. Consider the motion of a closed loop of homogeneous string in the absence of body forces. Let $\mathbf{r}(s, t) \in E^{3}$ (Euclidean 3-space) denote the position vector at time $t$ of the material point that has arclength coordinate $s$ in some natural state. We say that the string undergoes an axial motion if $\mathbf{r}(s, t)=\mathbf{p}(\lambda s+v t)$, where $\mathbf{p}: \mathbb{R} \rightarrow E^{3}$ is periodic with $\mathbf{p}^{\prime} \cdot \mathbf{p}^{\prime} \equiv 1$, and where $\lambda>0$ is the uniform stretch and $v \neq 0$ the constant speed of each particle. Note that $\mathbf{p}$ characterizes the shape of a fixed spatial configuration, through which the string steadily "flows." In a previous work [1], treating both inextensible chains and nonlinearly elastic strings, we showed that: (i) axial motions are independent of shape, i.e., local linear momentum balance is satisfied for any choice of $\mathbf{p}$ (assuming appropriate smoothness and periodicity); (ii) the most familiar axial motion-a circular shape rotating rigidly about its major axis of inertia-is energetically preferred over the others. More specifically, for constant energy ( $v=$ const.), the circular shape renders the magnitude of the total angular momentum a global maximum among all axial motions.

We view (ii) as a restricted stability result, which motivated the present study. In this paper we investigate the stability and bifurcation of the circular axial motion within a more general class of perturbations. Our approach to stability is based upon a well known idea (going back at least to Routh [2]) that has recently been formalized in a modern context, cf. [3, 4, 5]. Specifically, we seek local minimizers of the total energy subject to the constraint of constant global angular momentum. Once the existence of such minimizers has been established, it is relatively straightforward to demonstrate Liapunov stability of motion, employing an augmented energy functional as a Liapunov function. Strictly speaking, we establish orbital Liapunov stability, in the sense that steadily rotating motions (in the material description) are given only to within an arbitrary temporal phase.

The outline of this paper is as follows. In Sec. 2 we pose our minimization problem in the language of the calculus of variations with constraints (e.g., cf. [6]). For both inextensible chains and nonlinearly elastic strings, we demonstrate that the circular axial motion corresponds to a critical point of the appropriate augmented energy functional. In Sec. 3 we show that an inextensible circular loop is unconditionally 
(for all nonzero angular speeds) orbitally stable. In Sec. 4 we investigate the stability of nonlinearly elastic loops, choosing the magnitude of the angular momentum as the control parameter. In this case, we classify strings as being either stiff or soft, according to a convenient and physically relevant criterion. We demonstrate that stiff strings are unconditionally orbitally stable, while soft strings are orbitally stable only if the control parameter is below a critical value. In Sec. 5 we show that a soft elastic loop will (generically) admit an $O(2)$-symmetry-breaking branch of bifurcating solutions, with bifurcation occurring at the critical value of the control parameter. We demonstrate that the generality of our model (within the confines of physically reasonable constitutive laws) permits a rich variety of bifurcation phenomena corresponding to noncircular solutions.

In spite of its elegance and conceptual simplicity, our problem has apparently not been analyzed before. Consequently, our results are new. There are several plausible explanations for its absence from the literature: (i) the effects of gravity render experimental observation of the phenomena difficult; (ii) a systematic approach to the stability analysis of steadily rotating continuum problems has only recently been sorted out [3]; (iii) neither inextensible loops nor stiff elastic loops yield bifurcating solutions. Of these, (iii) is perhaps the most likely. Indeed, the linear elastic string (Hooke's law) of classical mechanics [7] is stiff according to our classification. In particular, bifurcation cannot be detected for either inextensible or linearly elastic models, the exclusive use of which is common even today.

2. Formulation and trivial solution. Consider a closed loop of string of total length $2 \pi$ in some homogeneous, tension-free (but otherwise arbitrary) reference configuration. We identify a material point by its arclength coordinate $s$ in that natural state. Let $\mathbf{r}(s, t) \in E^{3}$ denote the position vector of particle $s \in \mathbb{R}_{2 \pi} \equiv \mathbb{R}(\bmod 2 \pi)$ at time $t \in \mathbb{R}$, as measured from a fixed origin. The local form of linear momentum balance is expressed by

$$
\begin{gathered}
\mathbf{r}_{t}=\mathbf{v} \\
\mathbf{v}_{t}=(T \mathbf{r})_{s},
\end{gathered}
$$

where $\mathbf{v}$ is the velocity field, $T$ is the tension in the string, and $(\cdot)_{t} \equiv \partial(\cdot) / \partial t$, etc. If the string is inextensible, we supplement (2.1) with

$$
\left|\mathbf{r}_{s}\right|^{2} \equiv 1
$$

where $|\mathbf{a}|^{2} \equiv \mathbf{a} \cdot \mathbf{a}$ for all $\mathbf{a} \in E^{3}$. On the other hand, a hyperelastic string is characterized by a constitutive law of the form

$$
T=W^{\prime}\left(\left|\mathbf{r}_{s}\right|\right)
$$

where $W$ denotes the free-energy density. We assume that $W:(0, \infty) \rightarrow \mathbb{R}$ is twice continuously differentiable, with $W^{\prime}(1)=0$ and $W^{\prime \prime}>0$ on $(0, \infty)$.

We seek motions that render the total energy of the string stationary, under the constraint of constant angular momentum. Let $\{\mathbf{i}, \mathbf{j}, \mathbf{k}\}$ denote the standard orthonormal basis for $E^{3}$. Without loss of generality, we prescribe the angular momentum parallel to $\mathbf{k}$. Accordingly, for an inextensible string we seek critical points 
of the functional

$$
\begin{aligned}
\mathscr{H}_{1}(\mathbf{v}, \mathbf{r}, \boldsymbol{\omega}, T) \equiv & \int_{0}^{2 \pi}\left[|\mathbf{v}|^{2} / 2+T\left(\left|\mathbf{r}_{s}\right|^{2}-1\right) / 2\right] d s \\
& -\boldsymbol{\omega} \cdot\left[\int_{0}^{2 \pi} \mathbf{r} \times \mathbf{v} d s-2 \pi \mu \mathbf{k}\right],
\end{aligned}
$$

where $T: \mathbb{R}_{2 \pi} \times \mathbb{R} \rightarrow \mathbb{R}$ and $\omega: \mathbb{R} \rightarrow E^{3}$ are Lagrange multipliers, and $\mu \neq 0$ is the prescribed magnitude of the (normalized) angular momentum, which we treat as a parameter. For hyperelastic strings, we seek extrema of

$$
\begin{aligned}
\mathscr{H}_{2}(\mathbf{v}, \mathbf{r}, \boldsymbol{\omega}) \equiv & \int_{0}^{2 \pi}\left[|\mathbf{v}|^{2} / 2+W\left(\left|\mathbf{r}_{s}\right|\right)\right] d s \\
& -\boldsymbol{\omega} \cdot\left[\int_{0}^{2 \pi} \mathbf{r} \times \mathbf{v} d s-2 \pi \mu \mathbf{k}\right] .
\end{aligned}
$$

In seeking minima, we view $\mathscr{H}_{1}$ and $\mathscr{H}_{2}$ as functionals on phase space, viz., the set of all sufficiently smooth (cf. Sec. 3) maps $(\mathbf{v}(\cdot, t), \mathbf{r}(\cdot, t)): \mathbb{R}_{2 \pi} \rightarrow E^{3} \times E^{3}$. We first carry out the formal procedures of the calculus of variations necessary for obtaining a smooth minimum. The first variation of $\mathscr{H}_{1}$ is given by

$$
\begin{aligned}
\delta \mathscr{H}_{1} & \left.\equiv \frac{d}{d \alpha} \mathscr{H}_{1}(\mathbf{v}+\alpha \boldsymbol{\zeta}, \mathbf{r}+\boldsymbol{\eta}, \boldsymbol{\omega}, T)\right|_{\alpha=0} \\
& =\int_{0}^{2 \pi}\left[\mathbf{v} \cdot \boldsymbol{\zeta}+T\left(\mathbf{r}_{s} \cdot \boldsymbol{\eta}_{s}\right)-\boldsymbol{\omega} \cdot(\boldsymbol{\eta} \times \mathbf{v}+\mathbf{r} \times \boldsymbol{\zeta})\right] d s .
\end{aligned}
$$

Integration by parts and standard arguments yield the Euler-Lagrange equations:

$$
\begin{gathered}
\mathbf{v}=\boldsymbol{\omega} \times \mathbf{r} \\
\left(T \mathbf{r}_{s}\right)_{s}=\boldsymbol{\omega} \times \mathbf{v},
\end{gathered}
$$

subject to the constraints $(2.2)$ and

$$
\int_{0}^{2 \pi} \mathbf{r} \times \mathbf{v} d s=2 \pi \mu \mathbf{k}
$$

Equation (2.6a) implies that the extremizers of $\mathscr{H}_{1}$ are rigid rotations, characterized by angular velocity $\omega$. Note that (2.6) corresponds to (2.1) when the latter is specialized to rigid rotations. The tension $T$ enforces the inextensibility constraint (2.2), and condition (2.7) prescribes the total angular momentum.

The absence of external forces in our problem implies that the total linear momentum of the chain is conserved. Consequently, the mass center translates at a constant velocity, which, without loss of generality, we take to be zero. Accordingly, we append to (2.2), (2.6), and (2.7) the side condition

$$
\int_{0}^{2 \pi} \mathbf{r} d s=0
$$

which fixes the mass center at the origin. 
We seek a solution of the form

$$
\boldsymbol{\omega}=\omega \mathbf{k}, \quad \mathbf{r}=\mathbf{e}(s+\omega t)
$$

where

$$
\mathbf{e}(\nu) \equiv \cos (\nu) \mathbf{i}+\sin (\nu) \mathbf{j},
$$

corresponding to a circular steadily rotating solution. The substitution of (2.9) into (2.6)-(2.8) yields

$$
\begin{aligned}
\boldsymbol{\omega}_{e} & =\mu \mathbf{k}, \quad T_{e}=\mu^{2}, \\
\mathbf{r}_{e} & =\mathbf{e}(s+\mu t) \quad \text { and } \quad \mathbf{v}_{e}=\mu \mathbf{e}^{\prime}(s+\mu t),
\end{aligned}
$$

which is both a critical point of (2.4) and a solution of (2.1) and (2.2).

Similarly, the Euler-Lagrange equations for (2.5) are

$$
\begin{gathered}
\mathbf{v}=\boldsymbol{\omega} \times \mathbf{r} \\
\left(\left[W^{\prime}\left(\left|\mathbf{r}_{s}\right|\right) /\left|\mathbf{r}_{s}\right|\right] \mathbf{r}_{s}\right)_{s}=\boldsymbol{\omega} \times \mathbf{v},
\end{gathered}
$$

subject to (2.7) and (2.8). Since the string is extensible in this case, we seek a solution of the form $\boldsymbol{\omega}=\omega \mathbf{k}$ and $\mathbf{r}=\lambda \mathbf{e}(s+\omega t) \quad(\lambda>0)$, the substitution of which into (2.7) and (2.11) gives

$$
\omega=\mu / \lambda^{2}
$$

and

$$
\lambda^{3} W^{\prime}(\lambda)=\mu^{2}
$$

respectively.

The properties of $W$ insure that $\lambda \mapsto \lambda^{3} W^{\prime}(\lambda)$ is a monotonically increasing function that maps $(1, \infty)$ onto $(0, \infty)$. Hence, we can invert $(2.12)$ globally to obtain $\lambda$ as a unique function of $\mu^{2}$, denoted $\lambda=\tilde{\lambda}\left(\mu^{2}\right)$, where $\tilde{\lambda}:(0, \infty) \rightarrow(1, \infty)$ is $C^{1}$ and monotonically increasing. Hence,

$$
\begin{gathered}
\tilde{\boldsymbol{\omega}}_{e}=\tilde{\omega}(\mu) \mathbf{k}, \\
\tilde{\mathbf{r}}_{e}=\tilde{\lambda}\left(\mu^{2}\right) \mathbf{e}(s+\tilde{\omega}(\mu) t),
\end{gathered}
$$

and

$$
\tilde{\mathbf{v}}_{e} \equiv \tilde{\omega}(\mu) \tilde{\lambda}\left(\mu^{2}\right) \mathbf{e}^{\prime}(s+\tilde{\omega}(\mu) t), \quad \text { where } \tilde{\omega}(\mu) \equiv \mu /\left[\tilde{\lambda}\left(\mu^{2}\right)\right]^{2},
$$

is both a critical point of (2.5) and a solution of (2.1) and (2.3).

REMARK 2.14. Recall that only rigid rotations qualify as critical points of our energy functionals, cf. (2.6) and (2.11). It is easy to show that of the uncountably many axial motions (discussed in Sec. 1), only the circular ones are rigid. That is, the noncircular axial motions do not render the total energy stationary (for constant angular momentum). While this fact and the results of [1] certainly suggest that the noncircular axial motions are unstable, we merely conclude here that their stability cannot be analyzed by the approach employed in this work. 
3. Stability of inextensible loops. In this section we analyze the stability of the circular configuration for inextensible chains. The basic idea is to employ $\mathscr{H}_{1}$ as a Liapunov function. Our first step is to examine the second variation of $\mathscr{H}_{1}$ evaluated at the critical point $(2.10)$ :

$$
\begin{aligned}
\delta^{2} \mathscr{H}_{1}^{e}(\boldsymbol{\zeta}, \boldsymbol{\eta}, \mu) & \left.\equiv \frac{d^{2}}{d \alpha^{2}} \mathscr{H}_{1}\left(\mathbf{v}_{e}+\alpha \boldsymbol{\zeta}, \mathbf{r}_{e}+\alpha \boldsymbol{\eta}, \mu \mathbf{k}, \mu^{2}\right)\right|_{\alpha=0} \\
& =\int_{0}^{2 \pi}\left[|\boldsymbol{\zeta}|^{2}+\mu^{2}\left|\boldsymbol{\eta}_{s}\right|^{2}-2 \mu \mathbf{k} \cdot(\boldsymbol{\eta} \times \boldsymbol{\zeta})\right] d s .
\end{aligned}
$$

We say that the pair $(\boldsymbol{\zeta}, \boldsymbol{\eta})$ is an admissible variation if, for all $t \in \mathbb{R}, s \mapsto(\boldsymbol{\zeta}(s, t)$, $\boldsymbol{\eta}(s, t))$ is $2 \pi$-periodic, $\boldsymbol{\zeta}(\cdot, t)$ is absolutely continuous (continuous and possesses a classical derivative a.e. $), \boldsymbol{\eta}(\cdot, t)$ is of class $C^{1}$ with $\boldsymbol{\eta}_{s}(\cdot, t)$ absolutely continuous, and the following conditions (linearized constraints) hold:

$$
\begin{gathered}
\mathbf{e}^{\prime}(s+\mu t) \cdot \boldsymbol{\eta}_{s} \equiv 0, \\
\int_{0}^{2 \pi}\left[\mu \boldsymbol{\eta} \times \mathbf{e}^{\prime}(s+\mu t)+\mathbf{e}(s+\mu t) \times \zeta\right] d s=\mathbf{0},
\end{gathered}
$$

and

$$
\int_{0}^{2 \pi} \boldsymbol{\eta} d s=\mathbf{0} \quad \forall t \in \mathbb{R} .
$$

We first demonstrate that the second variation is positive semi-definite.

LemMA 3.3. $\delta^{2} \mathscr{H}_{1}^{e}(\boldsymbol{\zeta}, \boldsymbol{\eta}, \mu) \geq 0$ for all admissible variations.

Proof. From the basic property of the Rayleigh quotient, we have the Poincare inequality

$$
\int_{0}^{2 \pi}\left|\boldsymbol{\eta}_{s}\right|^{2} d s \geq \sigma^{2} \int_{0}^{2 \pi}|\boldsymbol{\eta}|^{2} d s
$$

where $\sigma^{2}$ is the minimum eigenvalue of

$$
-\boldsymbol{\eta}_{s s}=\sigma^{2} \boldsymbol{\eta}
$$

subject to (3.2) and $2 \pi$-periodicity in $s$. A simple calculation with the aid of $(3.2 \mathrm{c})$ shows that $\sigma^{2}=1$. Thus,

$$
\begin{aligned}
\delta^{2} \mathscr{H}_{1}^{e}(\boldsymbol{\zeta}, \boldsymbol{\eta}, \mu) & \geq \int_{0}^{2 \pi}\left[|\boldsymbol{\zeta}|^{2}+\mu^{2}|\boldsymbol{\eta}|^{2}-2 \mu \mathbf{k} \cdot(\boldsymbol{\eta} \times \boldsymbol{\zeta})\right] d s \\
& =\int_{0}^{2 \pi}\left[\left(\zeta_{1}+\mu \eta_{2}\right)^{2}+\left(\zeta_{2}-\mu \eta_{1}\right)^{2}+\mu^{2}\left(\eta_{3}\right)^{2}+\left(\zeta_{3}\right)^{2}\right] d s \geq 0,
\end{aligned}
$$

where $\boldsymbol{\eta}=\eta_{1} \mathbf{i}+\eta_{2} \mathbf{j}+\eta_{3} \mathbf{k}$, etc.

On the other hand, we do not expect $\delta^{2} \mathscr{H}_{1}^{e}(\boldsymbol{\zeta}, \boldsymbol{\eta}, \mu)$ be positive definite. Indeed, the circular configuration is given only to within an arbitrary phase angle, i.e., $\mathbf{r}=$ $\mathbf{e}(s+\mu t+\psi)$ and $\mathbf{v}=\mu \mathbf{e}^{\prime}(s+\mu t+\psi)$ satisfy (2.2) and (2.6)-(2.8) (with $\boldsymbol{\omega}_{e}$ and $T_{e}$ as given by (2.10)) for all $\psi \in \mathbb{R}_{2 \pi}$. In other words, (2.10) is one member of a closed orbit of critical points, along which $\mathscr{H}_{1}$ is constant. Consequently, $\delta^{2} \mathscr{H}_{1}^{e}(\boldsymbol{\zeta}, \boldsymbol{\eta}, \mu)$ 
vanishes for all admissible variations tangential to the orbit, viz., for all (infinitesimal) rotations of the form

$$
\begin{gathered}
\boldsymbol{\eta}(s, t)=\alpha \mathbf{k} \times \mathbf{e}(s+\mu t+\psi) \\
=\alpha\left[-\sin \psi \mathbf{e}(s+\mu t)+\cos \psi \mathbf{e}^{\prime}(s+\mu t)\right], \\
\left.\boldsymbol{\zeta}(s, t)=\alpha \mathbf{k} \times \mu \mathbf{e}^{\prime}(s+\mu t+\psi)\right) \\
=-\alpha \mu\left[\cos \psi \mathbf{e}(s+\mu t)+\sin \psi \mathbf{e}^{\prime}(s+\mu t)\right] \quad \forall \alpha, \psi \in \mathbb{R} .
\end{gathered}
$$

Note that (3.6) at $\psi=\pi / 2$ is not allowed by (3.2a). Moreover, on physical grounds (as well as for mathematical convenience), we are not interested in the variations (3.6). Accordingly, we append to (3.2) the condition

$$
\int_{0}^{2 \pi}\left(\mathbf{e}^{\prime}(s+\mu t) \cdot \boldsymbol{\eta}-\mu \mathbf{e}(s+\mu t) \cdot \boldsymbol{\zeta}\right) d s=0,
$$

which insures that an admissible variation $(\boldsymbol{\eta}, \boldsymbol{\zeta})$ is orthogonal (in the $\mathbf{L}^{2}$ sense) to all variations of the form (3.6) at $\psi=0$.

Thus far, our development is independent of (3.2b). Moreover, we would like to eliminate (2.7) and (3.2b) from our forthcoming stability theorem. (Otherwise, we must restrict our analysis to the class of motions having the same angular momentum as $\left(\mathbf{v}_{e}, \mathbf{r}_{e}\right)$, viz., $\left.2 \pi \mu \mathbf{k}\right)$. Accordingly, we define

$$
\begin{array}{r}
D \equiv\left\{(\boldsymbol{\zeta}(\cdot, t), \boldsymbol{\eta}(\cdot, t)) \in C^{0}\left(\mathbb{R}_{2 \pi} ; E^{3}\right) \times C^{1}\left(\mathbb{R}_{2 \pi} ; E^{3}\right): \boldsymbol{\zeta}(\cdot, t) \text { and } \boldsymbol{\eta}_{s}(\cdot, t)\right. \text { are } \\
\text { absolutely continuous, and }(3.2 \mathrm{a}),(3.2 \mathrm{c}),(3.7) \text { hold } \forall t \in \mathbb{R}\},
\end{array}
$$

which is independent of $(3.2 b)$. We now conclude

LEMMA 3.8. $\delta^{2} \mathscr{H}_{1}^{e}(\boldsymbol{\zeta}, \boldsymbol{\eta}, \mu)>0$ for $\mu \neq 0$ and for all nonzero $(\boldsymbol{\zeta}, \boldsymbol{\eta}) \in D$. Moreover, the critical point $(2.10)$ is a relative minimum of the augmented energy functional (2.4).

Proof. For $(\boldsymbol{\zeta}, \boldsymbol{\eta}) \in D$, we have the Fourier-series representations

$$
\begin{gathered}
\boldsymbol{\eta}=\sum_{n=1}^{\infty}\left[\mathbf{a}_{n} \cos (n s)+\mathbf{b}_{n} \sin (n s)\right] \\
\boldsymbol{\zeta}=\mathbf{c}_{0}+\sum_{n=1}^{\infty}\left[\mathbf{c}_{n} \cos (n s)+\mathbf{d}_{n} \sin (n s)\right],
\end{gathered}
$$

the substitution of which into (3.1) yields

$$
\begin{aligned}
\delta^{2} \mathscr{H}_{1}^{e}(\boldsymbol{\zeta}, \boldsymbol{\eta}, \mu)=\pi & \left\{\sum _ { n = 1 } ^ { \infty } \left[\left(\mu a_{n 2}+c_{n 1}\right)^{2}+\left(\mu b_{n 2}+d_{n 1}\right)^{2}+\left(\mu a_{n 1}-c_{n 2}\right)^{2}\right.\right. \\
& +\left(\mu b_{n 1}-d_{n 2}\right)^{2}+\mu^{2}(n-1)\left(a_{n 1}^{2}+a_{n 2}^{2}+b_{n 1}^{2}+b_{n 2}^{2}\right) \\
& \left.\left.+\left(c_{n 3}^{2}+d_{n 3}^{2}\right)+\mu^{2} n\left(a_{n 3}^{2}+b_{n 3}^{2}\right)\right]+\left|\mathbf{c}_{0}\right|^{2}\right\}
\end{aligned}
$$


where $\mathbf{a}_{n}=a_{n 1} \mathbf{i}+a_{n 2} \mathbf{j}+a_{n 3} \mathbf{k}$, etc. Clearly, $\delta^{2} \mathscr{H}_{1}^{e}(\boldsymbol{\zeta}, \boldsymbol{\eta}, \mu) \geq 0$, with equality holding only if

i.e., only if

$$
\begin{gathered}
\mathbf{c}_{0}=\mathbf{a}_{n}=\mathbf{b}_{n}=\mathbf{c}_{n}=\mathbf{d}_{n}=\mathbf{0}, \quad \text { for } n=2,3, \ldots, \\
a_{13}=b_{13}=c_{13}=d_{13}=0, \quad c_{11}=-\mu a_{12}, \\
d_{11}=-\mu b_{12}, \quad c_{12}=\mu a_{11}, \quad \text { and } d_{12}=\mu b_{11},
\end{gathered}
$$

$$
\boldsymbol{\eta}=\left(c_{1} \mathbf{i}+c_{2} \mathbf{j}\right) \cos (s)+\left(c_{3} \mathbf{i}+c_{4} \mathbf{j}\right) \sin (s)
$$

and

$$
\boldsymbol{\zeta}=\mu \mathbf{k} \times \boldsymbol{\eta},
$$

where the $c_{i}, i=1,2 \ldots, 4$, are arbitrary functions of time. However, (3.2a) and (3.7) imply that $c_{i}=0, i=1, \ldots, 4$, for all $(\boldsymbol{\zeta}, \boldsymbol{\eta}) \in D$.

To see that $(2.10)$ is a relative minimum of $(2.4)$, we note that $(\mathbf{v}, \mathbf{r}) \mapsto$ $\mathscr{H}_{1}\left(\mathbf{v}, \mathbf{r}, \mu \mathbf{k}, \mu^{2}\right)$ is quadratic. Hence,

$$
\mathscr{H}_{1}\left(\mathbf{v}_{e}+\boldsymbol{\zeta}, \mathbf{r}_{e}+\boldsymbol{\eta}, \mu \mathbf{k}, \mu^{2}\right)-\mathscr{H}_{1}\left(\mathbf{v}_{e}, \mathbf{r}_{e}, \mu \mathbf{k}, \mu^{2}\right) \equiv \frac{1}{2} \delta^{2} \mathscr{H}_{1}{ }^{e}(\boldsymbol{\zeta}, \boldsymbol{\eta}, \mu),
$$

which is positive for all nonzero $(\boldsymbol{\zeta}, \boldsymbol{\eta}) \in D$.

We now establish orbital Liapunov stability of the motion (2.10). An easy calculation shows that $\mathscr{H}_{1}$ is constant along any classical solution of $(2.1),(2.2)$, and (2.8) (cf. Remark 3.16). Moreover, for any fixed value of $\mu \neq 0$, Lemma 3.8 insures that

$$
\|(\boldsymbol{\zeta}, \boldsymbol{\eta})\|^{2} \equiv \frac{1}{2} \delta^{2} \mathscr{H}_{1}^{e}(\boldsymbol{\zeta}, \boldsymbol{\eta}, \mu)
$$

defines a norm (equivalent to the usual $H^{1} \times L^{2}$ metric) on $D$. Suppose that $(\mathbf{v}, \mathbf{r}) \equiv$ $\left(\mathbf{v}_{e}, \mathbf{r}_{e}\right)+(\Delta \mathbf{v}, \Delta \mathbf{r})$ is a classical solution of $(2.1),(2.2)$, and (2.8). By virtue of (3.12), we have

$$
\begin{aligned}
\|(\Delta \mathbf{v}(t), \Delta \mathbf{r}(t))\|^{2} & =\mathscr{H}_{1}\left(\mathbf{v}(t), \mathbf{r}(t), \mu \mathbf{k}, \mu^{2}\right)-\mathscr{H}_{1}\left(\mathbf{v}_{e}, \mathbf{r}_{e}, \mu \mathbf{k}, \mu^{2}\right) \\
& =\mathscr{H}_{1}\left(\mathbf{v}(0), \mathbf{r}(0), \mu \mathbf{k}, \mu^{2}\right)-\mathscr{H}_{1}\left(\mathbf{v}_{e}, \mathbf{r}_{e}, \mu \mathbf{k}, \mu^{2}\right),
\end{aligned}
$$

where the last equality follows from the fact that $\mathscr{H}_{1}$ is a constant of the motion. Then (3.12)-(3.14) yield

TheOREM 3.15. The motion (2.10) is orbitally Liapunov stable, i.e., given an $\varepsilon>0$, then $\left\|(\mathbf{v}(0), \mathbf{r}(0))-\left(\mathbf{v}_{e}, \mathbf{r}_{e}\right)\right\|^{2}<\varepsilon$ implies that

$$
\left\|(\mathbf{v}(t), \mathbf{r}(t))-\left(\mathbf{v}_{e}, \mathbf{r}_{e}\right)\right\|^{2}=\left|\mathscr{H}_{1}\left(\mathbf{v}(0), \mathbf{r}(0), \mu \mathbf{k}, \mu^{2}\right)-\mathscr{H}_{1}\left(\mathbf{v}_{e}, \mathbf{r}_{e}, \mu \mathbf{k}, \mu^{2}\right)\right|<\varepsilon .
$$

REMARK 3.16. In developing our stability theorem, we have tacitly assumed that $C^{1}$ solutions of the initial-value problem for (2.1) and (2.2) exist for all time, which may not be the case. Indeed, we know of no such global existence theorems; (2.1) and (2.2) may conceivably admit solutions with shocks for smooth initial data. Nonetheless, our results are rigorous for all times during which $C^{1}$ solutions exist.

Finally, it is easy to show that the linearization of (2.6) about the trivial solution (2.10) ultimately leads to the equation

$$
\boldsymbol{\eta}_{s s}+\boldsymbol{\eta}-(\mathbf{k} \cdot \boldsymbol{\eta}) \mathbf{k}=\mathbf{0},
$$


which is independent of $\mu$. Thus, we conclude

THEOREM 3.17. With $\mu=\omega$ as a control parameter, there is no bifurcation from the circular configuration (2.10) to other steadily rotating states.

4. Stability of hyperelastic loops. We consider the stability of the circular configuration (2.13) in this section, employing the energy functional (2.5) as a Liapunov function. The second variation of $\mathscr{H}_{2}$ evaluated at (2.13) is given by

$$
\begin{aligned}
\delta^{2} \mathscr{H}_{2}^{e}(\boldsymbol{\zeta}, \boldsymbol{\eta}, \mu) \equiv & \left.\frac{d^{2}}{d \alpha^{2}} \mathscr{H}_{2}\left(\tilde{\mathbf{v}}_{e}+\alpha \boldsymbol{\zeta}, \tilde{\mathbf{r}}_{e}+\alpha \boldsymbol{\eta}, \tilde{\boldsymbol{\omega}}_{e}\right)\right|_{\alpha=0} \\
= & \int_{0}^{2 \pi}\left\{|\boldsymbol{\zeta}|^{2}+\left[W^{\prime}\left(\tilde{\lambda}\left(\mu^{2}\right)\right) / \tilde{\lambda}\left(\mu^{2}\right)\right]\left|\boldsymbol{\eta}_{s}\right|^{2}\right. \\
& +\left[W^{\prime \prime}\left(\tilde{\lambda}\left(\mu^{2}\right)\right)-W^{\prime}\left(\tilde{\lambda}\left(\mu^{2}\right)\right) / \tilde{\lambda}\left(\mu^{2}\right)\right]\left(\mathbf{e}^{\prime} \cdot \boldsymbol{\eta}_{s}\right)^{2} \\
& -2 \tilde{\omega}(\mu) \mathbf{k} \cdot(\boldsymbol{\eta} \times \boldsymbol{\zeta})\} d s .
\end{aligned}
$$

We place the same smoothness requirements on an admissible variation $(\boldsymbol{\zeta}, \boldsymbol{\eta})$ as in Sec. 3. In this case, the linearized constraints are

$$
\int_{0}^{2 \pi}\left[\tilde{\omega}(\mu) \boldsymbol{\eta} \times \mathbf{e}^{\prime}(s+\tilde{\omega}(\mu) t)+\mathbf{e}(s+\tilde{\omega}(\mu) t) \times \zeta\right] d s=\mathbf{0},
$$

and

$$
\int_{0}^{2 \pi} \boldsymbol{\eta} d s=\mathbf{0}
$$

The third term of the integrand in (4.1), which does not appear in (3.1), plays a crucial role in our forthcoming analysis of stability and bifurcation. Accordingly, we find it convenient to make the following classification.

Definition 4.2. A hyperelastic string, characterized by the free-energy function $W$, is said to be stiff if

$$
\frac{d}{d \nu}\left[W^{\prime}(\nu) / \nu\right]>0 \quad \forall \nu \in(1, \infty) .
$$

Otherwise, the string is said to be soft.

Remark 4.3. We can interpret Definition 4.2 geometrically by referring to the graph of $W^{\prime}$ (the constitutive law $T=W^{\prime}(\lambda)$ vs. $\lambda$ ). A string is stiff if the secant $W^{\prime}(\lambda) / \lambda$ is always less than the tangent modulus of elasticity, $W^{\prime \prime}(\lambda)$. Typical constitutive laws for stiff and soft elastic strings are illustrated in Fig. 1. Note that our previous restrictions on $W$ (cf. Sec. 2) insure that even soft strings have a positive tangent modulus, i.e., $W^{\prime \prime}(\nu)>0 \quad \forall \nu \in(0, \infty)$.

We first show that all strings are "initially stiff."

Lemma 4.4. For all soft strings, there is a number $\lambda_{*}>1$ such that

$$
\frac{d}{d \nu}\left[W^{\prime}(\nu) / \nu\right]>0 \quad \forall \nu \in\left[1, \lambda_{*}\right), \quad \text { and }\left.\quad \frac{d}{d \nu}\left[W^{\prime}(\nu) / \nu\right]\right|_{\nu=\lambda_{*}}=0 .
$$

Proof. Our restrictions on $W$ imply that $\nu \mapsto g(\nu) \equiv\left[W^{\prime \prime}(\nu) / \nu-W^{\prime}(\nu) / \nu^{2}\right]$ is continuous on $(0, \infty)$ with $g(1)=W^{\prime \prime}(1)>0$. Thus, $g>0$ on some open interval 


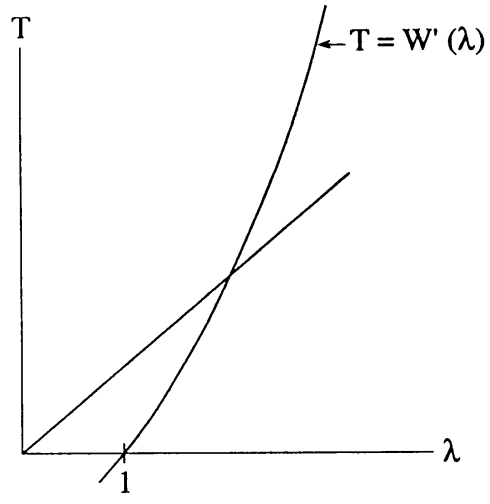

(a) stiff

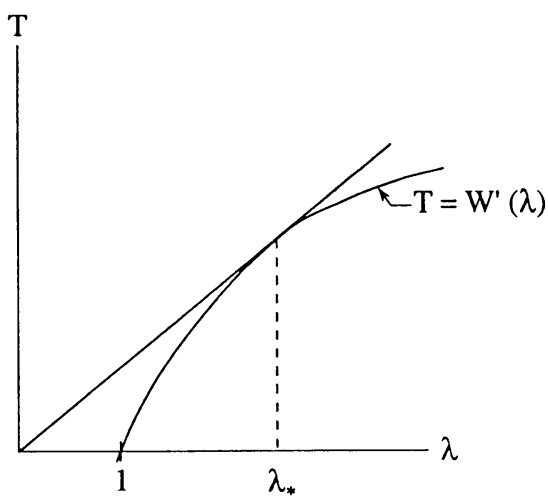

(b) soft

FIG. 1. Typical constitutive relations for elastic strings.

containing $\nu=1$. Definition 4.2 implies that $g$ has at least one zero on $(1, \infty)$, the smallest of which we denote by $\lambda_{*}$, cf. Fig. $1 \mathrm{~b}$.

Before presenting our stability theorem, we note that, like (2.10), the critical point (2.13) is given only to within an arbitrary phase angle. Thus, (4.1) vanishes for all variations of the form

$$
\begin{aligned}
\boldsymbol{\eta}(s, t) & =\alpha \mathbf{k} \times \mathbf{e}(s+\tilde{\omega}(\mu) t+\psi) \\
& =\alpha\left[-\sin \psi \mathbf{e}(s+\tilde{\omega}(\mu) t)+\cos \psi \mathbf{e}^{\prime}(s+\tilde{\omega}(\mu) t)\right], \\
\zeta(s, t) & \left.=\alpha \mathbf{k} \times \mu \mathbf{e}^{\prime}(s+\tilde{\omega}(\mu) t+\psi)\right) \\
& =-\alpha \tilde{\omega}(\mu)\left[\cos \psi \mathbf{e}(s+\tilde{\omega}(\mu) t)+\sin \psi \mathbf{e}^{\prime}(s+\tilde{\omega}(\mu) t)\right] . \quad \forall a, \psi \in \mathbb{R} .
\end{aligned}
$$

In this case, $(4.5)$ at $\psi=\pi / 2$ is ruled out by $(4.2 \mathrm{a})$. However, we would like to eliminate both (2.7) and (4.2a) from our forthcoming stability theorem, for the same reasons given in Sec. 3, cf. (3.2b) and Theorem 3.15. Since the string is extensible, we have no analogue of (3.2a) in this case. Hence, without (4.2a), we must now append two orthogonality conditions to (4.2b) (cf. (3.7)):

$$
\int_{0}^{2 \pi}\left[\mathbf{e}^{\prime}(s+\tilde{\omega}(\mu) t) \cdot \boldsymbol{\eta}-\tilde{\omega}(\mu) \mathbf{e}(s+\tilde{\omega}(\mu) t) \cdot \zeta\right] d s=0,
$$

and

$$
\int_{0}^{2 \pi}\left[\mathbf{e}(s+\tilde{\omega}(\mu) t) \cdot \boldsymbol{\eta}+\tilde{\omega}(\mu) \mathbf{e}^{\prime}(s+\tilde{\omega}(\mu) t) \cdot \boldsymbol{\zeta}\right] d s=0,
$$

which insure that a variation $(\boldsymbol{\zeta}, \boldsymbol{\eta})$ is orthogonal to all variations of the form (4.5). We define

$$
\begin{aligned}
D \equiv\{(\boldsymbol{\zeta}(\cdot, t), \boldsymbol{\eta}(\cdot, t)) \in & C^{0}\left(\mathbb{R}_{2 \pi} ; E^{3}\right) \times C^{1}\left(\mathbb{R}_{2 \pi} ; E^{3}\right): \zeta(\cdot, t) \text { and } \boldsymbol{\eta}_{s}(\cdot, t) \text { are } \\
& \text { absolutely continuous, and }(4.2 \mathrm{~b}),(4.6) \text { hold } \forall t \in \mathbb{R}\} .
\end{aligned}
$$

We now conclude

LEMMA 4.7. The following claims hold for all stiff strings with $\mu \neq 0$ and for all soft strings with $0<\mu^{2}<\mu_{*}^{2} \equiv \lambda_{*}^{3} W^{\prime}\left(\lambda_{*}\right)$. The second variation is positive definite, 
i.e., $\delta^{2} \mathscr{H}_{2}^{e}(\boldsymbol{\zeta}, \boldsymbol{\eta}, \mu)>0$ for all nonzero $(\boldsymbol{\zeta}, \boldsymbol{\eta}) \in D$, and the critical point $(2.13)$ is a (weak) relative minimizer of the augmented energy functional (2.5).

Proof. By virtue of (2.12), (4.1), and Definition 4.2 (or Lemma 4.4), it follows that

$$
\left.\delta^{2} \mathscr{H}_{2}^{e}(\boldsymbol{\zeta}, \boldsymbol{\eta}, \mu) \geq Q(\boldsymbol{\zeta}, \boldsymbol{\eta}, \mu) \equiv \int_{0}^{2 \pi}\left\{|\boldsymbol{\zeta}|^{2}+[\tilde{\omega}(\mu)]^{2}\right)\left|\boldsymbol{\eta}_{s}\right|^{2}-2 \tilde{\omega}(\mu) \mathbf{k} \cdot(\boldsymbol{\eta} \times \boldsymbol{\zeta})\right\} d s .
$$

Note that $Q(\boldsymbol{\zeta}, \boldsymbol{\eta}, \mu)$ is identical to $\delta^{2} \mathscr{H}_{1}^{e}(\boldsymbol{\zeta}, \boldsymbol{\eta}, \mu)$, except that $\tilde{\omega}(\mu)$ is now in place of $\mu$, cf. (3.1). Hence, from the proof of Lemma 3.3, we conclude that $Q$ is positive semi-definite. To show definiteness, we emulate the proof of Lemma (3.8) to conclude that $Q(\boldsymbol{\zeta}, \boldsymbol{\eta}, \mu)=0$ only if $(\boldsymbol{\zeta}, \boldsymbol{\eta})$ is of the form (3.11). In this case, conditions (4.6) imply that $c_{i}=0, i=1, \ldots, 4$.

We now demonstrate that $(2.13)$ is a weak relative minimum of (2.5), i.e., a relative minimum with respect to the usual $C^{0} \times C^{1}$ topology induced by

$$
\|(\boldsymbol{\zeta}, \boldsymbol{\eta})\|_{C^{0} \times C^{1}} \equiv \max _{s \in \mathbb{R}_{2 \pi}}|\boldsymbol{\zeta}|+\max _{s \in \mathbb{R}_{2 \pi}}|\boldsymbol{\eta}|+\max _{s \in \mathbb{R}_{2 \pi}}\left|\boldsymbol{\eta}_{s}\right| .
$$

First we define

$$
\begin{aligned}
\delta^{2} \mathscr{H}_{2}(\mathbf{v}, \mathbf{r}, \mu ; \boldsymbol{\zeta}, \boldsymbol{\eta}) \equiv & \left.\frac{d^{2}}{d \alpha^{2}} \mathscr{H}_{2}\left(\mathbf{v}+\alpha \boldsymbol{\zeta}, \mathbf{r}+\alpha \boldsymbol{\eta}, \tilde{\boldsymbol{\omega}}_{e}\right)\right|_{r=0} \\
= & \int_{0}^{2 \pi}\left\{|\boldsymbol{\zeta}|^{2}+\left[W^{\prime}\left(\left|\mathbf{r}_{s}\right|\right) /\left|\mathbf{r}_{s}\right|\right]\left|\boldsymbol{\eta}_{s}\right|^{2}\right. \\
& \left.+\left[W^{\prime \prime}\left(\left|\mathbf{r}_{s}\right|\right)-W^{\prime}\left(\left|\mathbf{r}_{s}\right|\right) /\left|\mathbf{r}_{s}\right|\right]\left(\mathbf{r}_{s} \cdot \boldsymbol{\eta}_{s}\right)^{2} /\left|\mathbf{r}_{s}\right|^{2}-2 \tilde{\omega}(\mu) \mathbf{k} \cdot \boldsymbol{\eta} \times \boldsymbol{\zeta}\right\} d s .
\end{aligned}
$$

By (2.13) and Taylor's theorem, there is a number $0<\tau<1$ such that

$$
\begin{aligned}
\mathscr{H}_{2}\left(\tilde{\mathbf{v}}_{e}\right. & \left.+\boldsymbol{\zeta}, \tilde{\mathbf{v}}_{e}+\boldsymbol{\eta}, \tilde{\boldsymbol{\omega}}_{e}\right)-\mathscr{H}_{2}\left(\tilde{\mathbf{v}}_{e}, \tilde{\mathbf{r}}_{e}, \tilde{\boldsymbol{\omega}}_{e}\right) \\
& =\frac{1}{2} \delta^{2} \mathscr{H}_{2}\left(\tilde{\mathbf{v}}_{e}+\tau \boldsymbol{\zeta}, \tilde{\mathbf{r}}_{e}+\tau \boldsymbol{\eta}, \mu ; \boldsymbol{\zeta}, \boldsymbol{\eta}\right) \\
& \geq \frac{1}{2} \int_{0}^{2 \pi}\left[|\boldsymbol{\zeta}|^{2}+\left[W^{\prime}\left(\left|\tilde{\lambda}\left(\mu^{2}\right) \mathbf{e}^{\prime}+\tau \boldsymbol{\eta}_{s}\right|\right) /\left|\tilde{\lambda}\left(\mu^{2}\right) \mathbf{e}^{\prime}+\tau \boldsymbol{\eta}_{s}\right|\right]\left|\boldsymbol{\eta}_{s}\right|^{2}-2 \tilde{\omega}(\mu) \mathbf{k} \cdot(\boldsymbol{\eta} \times \boldsymbol{\zeta})\right] d s,
\end{aligned}
$$

for $\|(\boldsymbol{\zeta}, \boldsymbol{\eta})\|_{C^{0} \times C^{1}}$ sufficiently small, where the last inequality follows from Definition 4.2 (or Lemma 4.4) and (4.10). Moreover, (2.12) and the smoothness of $W$ insure that for $\varepsilon>0$, we have

$$
\begin{aligned}
& \left.\left|\int_{0}^{2 \pi}\left[W^{\prime}\left(\left|\tilde{\lambda}\left(\mu^{2}\right) \mathbf{e}^{\prime}+\tau \boldsymbol{\eta}_{s}\right|\right) /\left|\tilde{\lambda}\left(\mu^{2}\right) \mathbf{e}^{\prime}+\tau \boldsymbol{\eta}_{s}\right|\right]\right| \boldsymbol{\eta}_{s}\right|^{2} d s-\int_{0}^{2 \pi}\left[\tilde{\omega}\left(\mu^{2}\right)\right]^{2}\left|\boldsymbol{\eta}_{s}\right|^{2} d s \mid \\
& \quad \leq \varepsilon \int_{0}^{2 \pi}\left|\boldsymbol{\eta}_{s}\right|^{2} d s \leq \varepsilon \kappa Q(\boldsymbol{\zeta}, \boldsymbol{\eta}, \mu),
\end{aligned}
$$

$\forall(\boldsymbol{\zeta}, \boldsymbol{\eta}) \in D$ with $\|(\boldsymbol{\zeta}, \boldsymbol{\eta})\|_{C^{0} \times C^{1}}$ sufficiently small, where $\kappa>0$ is a constant. Note that the left side of (4.12) is also the magnitude of the difference between $Q(\boldsymbol{\zeta}, \boldsymbol{\eta}, \mu)$ and two times the right side of (4.11). Thus, if $\varepsilon=1 / 2 \kappa$, say, then we have

$$
\mathscr{H}_{2}\left(\tilde{\mathbf{v}}_{e}+\boldsymbol{\zeta}, \tilde{\mathbf{r}}_{e}+\boldsymbol{\eta}, \tilde{\boldsymbol{\omega}}_{e}\right)-\mathscr{H}_{2}\left(\tilde{\mathbf{v}}_{e}, \tilde{\mathbf{r}}_{e}, \tilde{\boldsymbol{\omega}}_{e}\right) \geq Q(\boldsymbol{\zeta}, \boldsymbol{\eta}, \mu) / 4,
$$

which is positive for all nonzero $(\boldsymbol{\zeta}, \boldsymbol{\eta}) \in D$. 
The demonstration of orbital Liapunov stability of the motion (2.13) is nearly identical to the analysis of the motion (2.10) culminating in Theorem 3.15. Hence, we emphasize here only those aspects that differ from the development presented in Sec. 3. From the proof of Lemma 4.7, we see that

$$
\|(\boldsymbol{\zeta}, \boldsymbol{\eta})\|^{2} \equiv Q(\boldsymbol{\zeta}, \boldsymbol{\eta}, \mu)
$$

defines a norm on $D$. However, in this case we have no analogue of equality (3.13). Consequently, we must restrict the perturbations with the stronger topology of (4.9), as suggested by the conditions of inequality (4.13). Since $\mathscr{H}_{2}$ is a constant of the motion, (4.13) yields the following analogue of (3.14):

$$
\|(\Delta \mathbf{v}(t), \Delta \mathbf{r}(t))\|^{2} \leq \mathscr{H}_{2}\left(\mathbf{v}(0), \mathbf{r}(0), \tilde{\boldsymbol{\omega}}_{e}\right)-\mathscr{H}_{2}\left(\tilde{\mathbf{v}}_{e}, \tilde{\mathbf{r}}_{e}, \tilde{\boldsymbol{\omega}}_{e}\right),
$$

for all classical solutions $(\mathbf{v}, \mathbf{r}) \equiv\left(\tilde{\mathbf{v}}_{e}, \tilde{\mathbf{r}}_{e}\right)+(\Delta \mathbf{v}, \Delta \mathbf{r})$ of (2.1), (2.3), and (2.8). The smoothness of $W$ insures that $\mathscr{H}_{2}\left(\cdot, \cdot, \tilde{\boldsymbol{\omega}}_{e}\right)$ is continuous at $\left(\tilde{\mathbf{v}}_{e}, \tilde{\mathbf{r}}_{e}\right)$ with respect to the norm defined by (4.9), and thus, we conclude

THEOREM 4.14. Assume the hypotheses of Lemma 4.7. The motion (2.13) is orbitally Liapunov stable, i.e, given an $\varepsilon>0$, there exists a $\delta>0$ such that

$$
\left\|(\mathbf{v}(0), \mathbf{r}(0))-\left(\mathbf{v}_{e}, \mathbf{r}_{e}\right)\right\|_{C^{0} \times C^{1}}^{2}<\delta
$$

implies

$$
\left\|(\mathbf{v}(t), \mathbf{r}(t))-\left(\mathbf{v}_{e}, \mathbf{r}_{e}\right)\right\|^{2} \leq\left|\mathscr{H}_{2}\left(\mathbf{v}(0), \mathbf{r}(0), \tilde{\boldsymbol{\omega}}_{e}\right)-\mathscr{H}_{2}\left(\tilde{\mathbf{v}}_{e}, \tilde{\mathbf{r}}_{e}, \tilde{\boldsymbol{\omega}}_{e}\right)\right|<\varepsilon .
$$

5. Bifurcation analysis of elastic loops. In this section we consider the possibility of other steadily rotating solutions bifurcating from the circular one (2.13) for elastic strings. The governing equations are given by (2.11) subject to (2.7) and (2.8), which have (2.13) as a trivial solution. However, we find it convenient to reformulate the problem as follows. Referring to (2.9), we define

$$
\mathbf{e}_{1}(t) \equiv \mathbf{e}(\omega t) \quad \text { and } \quad \mathbf{e}_{2}(t) \equiv \mathbf{e}^{\prime}(\omega t),
$$

and seek planar solutions of the form

$$
\boldsymbol{\omega}=\omega \mathbf{k}, \quad \mathbf{r}(s, t)=\sum_{\alpha=1}^{2} u_{\alpha}(s) \mathbf{e}_{\alpha}(t) .
$$

Let $u$ denote the complex-valued function on $\mathbb{R}_{2 \pi}$ defined by

$$
u(s) \equiv u_{1}(s)+i u_{2}(s) .
$$

The substitution of (5.1) into (2.7) and (2.11), while employing (5.2) yields

$$
\left(q u^{\prime}\right)^{\prime}+4 \pi^{2} \mu^{2}\left(\int_{0}^{2 \pi}|u|^{2} d s\right)^{-2} u=0 \quad \text { on } \mathbb{R}_{2 \pi},
$$

where

$$
q(s) \equiv W^{\prime}\left(\left|u^{\prime}(s)\right|\right) /\left|u^{\prime}(s)\right|,
$$


and where

$$
\omega=2 \pi \mu\left(\int_{0}^{2 \pi}|u|^{2} d s\right)^{-1}
$$

which has been eliminated. Condition (2.8) is now expressed as

$$
\int_{0}^{2 \pi} u d s=0 .
$$

The trivial solution (2.13), corresponding to the circular configuration, is equivalent to

$$
u=\tilde{\lambda}\left(\mu^{2}\right) e^{i s},
$$

which is readily verified to be a solution of (5.3) satisfying (5.5).

A convenient componential form of $(5.3 \mathrm{a})$ results from the employment of polar coordinates $(r, \theta)$ via $u=r e^{i \theta}$, where $r(s)>0$ and $0 \leq \theta(s)<2 \pi$ on $\mathbb{R}_{2 \pi}$ :

$$
\begin{gathered}
\left(q r^{\prime}\right)^{\prime}+\left[4 \pi^{2} \mu^{2}\left(\int_{0}^{2 \pi} r^{2}, d s\right)^{-2}-q\left(\theta^{\prime}\right)^{2}\right] r=0 \\
\left(q \theta^{\prime} r^{2}\right)^{\prime}=0
\end{gathered}
$$

Of course (5.7b) admits the conserved quantity

$$
q \theta^{\prime} r^{2}=C \text {. }
$$

By virtue of $(5.3 \mathrm{~b})$, it follows that $(5.8)$ can be expressed as

$$
h\left(\theta^{\prime}, r, r^{\prime}\right)=0
$$

where $h: \mathbb{R}^{3} \rightarrow \mathbb{R}$ is $C^{1}$. At the trivial solution (5.6), we have

$$
h\left(1, \tilde{\lambda}\left(\mu^{2}\right), 0\right)=0 \text {, }
$$

and a straightforward calculation yields

$$
\frac{d h}{d \theta^{\prime}}\left(1, \tilde{\lambda}\left(\mu^{2}\right), 0\right)=W^{\prime \prime}\left(\tilde{\lambda}\left(\mu^{2}\right)\right)\left[\lambda\left(\mu^{2}\right)\right]^{2}>0 \text {. }
$$

Then by the implicit function theorem, there is a unique $C^{1}$ mapping $\chi: \mathbb{R}^{2} \rightarrow \mathbb{R}$ such that (5.8) is equivalent to

$$
\theta^{\prime}=\chi\left(r, r^{\prime}\right)
$$

for all $\left(r, r^{\prime}\right)$ in some sufficiently small neighborhood of $\left(\tilde{\lambda}\left(\mu^{2}\right), 0\right)$, with

$$
\chi\left(\tilde{\lambda}\left(\mu^{2}\right), 0\right)=1 .
$$

If we set $r=\tilde{\lambda}\left(\mu^{2}\right)+w$, then the left side of $(5.7 \mathrm{a})$, with the aid of $(5.3 \mathrm{~b})$ and (5.10), defines a mapping $(w, \lambda) \mapsto f(u, \lambda)$. Without loss of generality, we henceforth find it convenient to multiply the left side of $(5.7 \mathrm{a})$ by the positive quantity $\tilde{\lambda}\left(\mu^{2}\right) / W^{\prime}\left(\tilde{\lambda}\left(\mu^{2}\right)\right)$ in our definition of $f$. Let $C_{2 \pi}^{n}$ denote the space of all $n$-times 
continuously differentiable, real-valued functions on $\mathbb{R}_{2 \pi}$. We equip $C_{2 \pi}^{0}$ and $C_{2 \pi}^{2}$ with the usual norms

$$
\|w\|_{C^{0}}=\max _{s \in \mathbb{R}_{2 \pi}}|w(s)|
$$

and

$$
\|w\|_{C^{2}}=\|w\|_{C^{0}}+\left\|w^{\prime}\right\|_{C^{0}}+\left\|w^{\prime \prime}\right\|_{C^{0}},
$$

respectively. Let $\Omega$ denote some sufficiently small neighborhood of $w \equiv 0 \in C_{2 \pi}^{2}$. Clearly, $f: \Omega \times(0, \infty) \rightarrow C_{2 \pi}^{0}$, and the mapping $w \mapsto f\left(w, \mu^{2}\right)$ is readily shown to be continuously Fréchet differentiable on $\Omega$. We now represent our problem abstractly by

$$
f\left(w, \mu^{2}\right)=0
$$

where $f\left(0, \mu^{2}\right) \equiv 0 \quad \forall \mu^{2} \in(0, \infty)$.

Of course, a necessary condition for bifurcation is that the linearized problem,

$$
A\left(\mu^{2}\right) \phi=\left[D_{w} f\left(0, \mu^{2}\right)\right] \phi=0,
$$

admit a nontrivial solution pair $\left(\phi, \mu^{2}\right) \in C_{2 \pi}^{2} \times(0, \infty)$, where $D_{w} f$ denotes the Fréchet derivative of $f$ with respect to its first argument. A straightforward calculation employing (2.12) yields (5.13) explicitly:

$$
\phi^{\prime \prime}+\left[3+p\left(\tilde{\lambda}\left(\mu^{2}\right)\right)\right] \phi-\frac{2}{\pi} \int_{0}^{2 \pi} \phi d s=0, \quad \text { on } \mathbb{R}_{2 \pi},
$$

where

$$
p(\lambda) \equiv W^{\prime}(\lambda) / \lambda W^{\prime \prime}(\lambda)
$$

is the ratio of the secant to the tangent modulus, cf. Remark 4.3. Clearly, (5.14) admits nontrivial solutions on $\mathbb{R}_{2 \pi}$ iff the characteristic equation,

$$
p\left(\tilde{\lambda}\left(\mu^{2}\right)\right)=n^{2}-3
$$

has roots, denoted $\mu_{n}^{2}$, for $n \in \mathbb{N}$. Our previous assumptions on $W$ (cf. Sec. 2) insure that $p>0$, and by Definition 4.2, stiff strings are characterized by $p<1$. Thus, we have

THEOREM 5.17. The characteristic equation (5.16) has no roots for $n=1$. Moreover, if the string is stiff, then (5.16) has no roots $\forall n \in \mathbb{N}$. Thus, stiff strings do not admit bifurcating solutions from the circular configuration.

REMARK 5.18. In the context of our problem, Theorems 4.14 and 5.17 imply that a stiff string behaves much like an inextensible one, cf. Theorems 3.15 and 3.17.

The remainder of this section is devoted exclusively to soft strings. In this case, Lemma 4.4 and (5.15) imply that $p\left(\tilde{\lambda}\left(\mu^{2}\right)\right)=1$ (i.e., (5.16) for $n=2$ ) has at least one root, viz., $\mu^{2}=\mu_{*}^{2} \equiv \lambda_{*}^{3} W^{\prime}\left(\lambda_{*}\right)$. The existence of other roots depends crucially upon the behavior of $p$, which, in turn, depends upon that of $W, \mathrm{cf} .(5.15)$. At this level of generality, (5.16) can have no other roots, a finite number of additional roots, or infinitely many roots. Moreover, in the latter two cases, there can exist more than one root for fixed $n$. As an illustration, we depict several plausible constitutive 


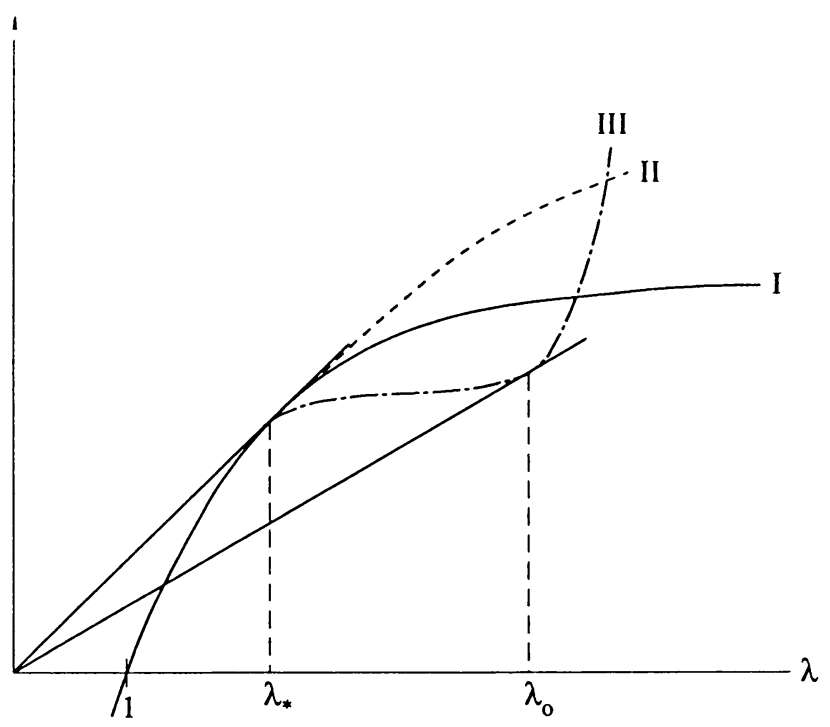

(a)

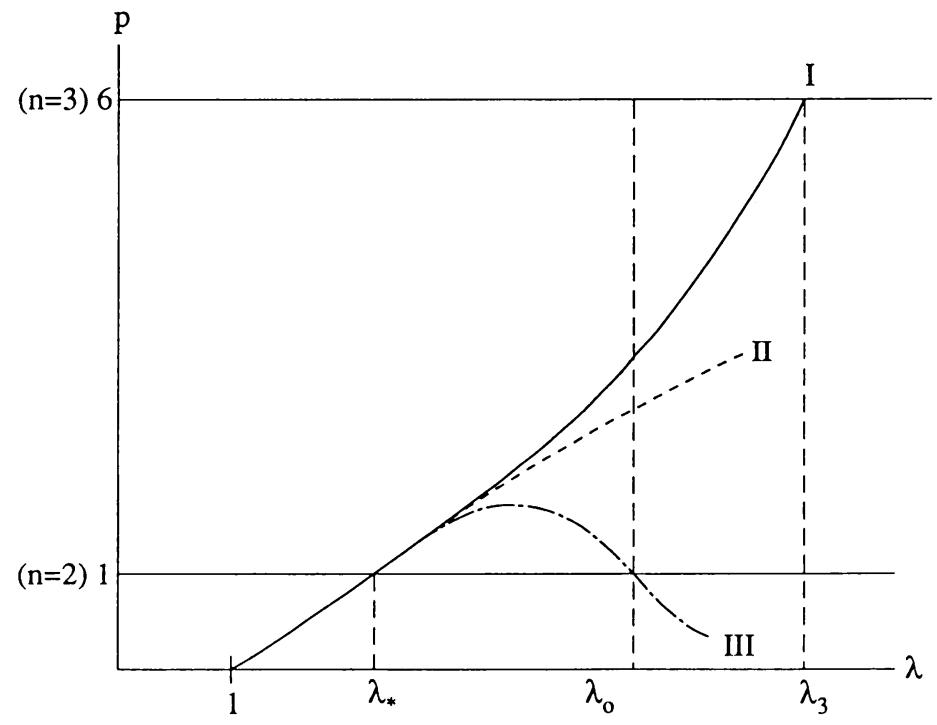

(b)

FIG. 2. Three exemplary constitutive laws for soft elastic strings and the corresponding graphs of Eq. (5.16).

relations in Fig. 2a, with the corresponding graphs of $p$ shown in Fig. 2b. We shall return to these after addressing sufficient conditions for bifurcation.

If $(5.16)$ has a root $\mu_{n}^{2}$, then the general solution of $(5.14)$ is

$$
\phi_{n}=A \sin (n s)+B \cos (n s)+C,
$$

where $A, B$, and $C$ are constants. However, $C$ is not arbitrary. Indeed, $C=0$ 
for $n>2$. For $n=2$, the linearization of (5.10) about $r \equiv \tilde{\lambda}\left(\mu_{2}^{2}\right)$ yields

$$
\theta^{\prime}=-\left[1+p\left(\tilde{\lambda}\left(\mu_{2}^{2}\right)\right)\right] \phi / \tilde{\lambda}\left(\mu_{2}^{2}\right) .
$$

The $2 \pi$-periodicity of $u=r e^{i \theta}$ demands that $\theta(s+2 \pi) \equiv 2 \pi+\theta(s)$, from which we deduce that $C=0$ (after integrating (5.20) via (5.19)). The constants $A$ and $B$ are arbitrary.

From (5.13) and (5.19), it follows that the kernel of $A\left(\mu_{n}^{2}\right)$ is two-dimensional, which ostensibly complicates a bifurcation analysis. However, this redundancy is a direct consequence of the $O(2)$ symmetry inherent in our problem, cf. [8]. Indeed, $w \mapsto f\left(w, \mu^{2}\right)$ is readily shown to be equivariant under the transformations $w(s) \rightarrow$ $w(s-\alpha), w(-s) \forall \alpha \in \mathbb{R}_{2 \pi}$, which generates a representation of $O(2)$ on $C_{2 \pi}^{n}$. We can employ well-known group theoretic tools to obtain bifurcation criteria for soft strings.

THEOREM 5.21. Let $\mu_{n}$ be a root of $(5.16)$, and consider the function $\sigma:(1, \infty) \mapsto \mathbb{R}$ defined by

$$
\sigma_{n}(\lambda) \equiv p(\lambda)+3-n^{2}
$$

Suppose that $\lambda_{n} \equiv \tilde{\lambda}\left(\mu_{n}\right)$ is the only zero of $\sigma_{n}$ on some sufficiently small neighborhood of $\lambda_{n}$, denoted $\left(\lambda_{n}-\varepsilon, \lambda_{n}+\varepsilon\right), \varepsilon>0$. If the sign of $\sigma_{n}$ on $\left(\lambda_{n}-\varepsilon, \lambda_{n}\right)$ is different from that on $\left(\lambda_{n}, \lambda_{n}+\varepsilon\right)$, then there is a local branch of nontrivial solutions $\sum_{n} \in \Omega \times(0, \infty)$, emanating from the trivial branch at $\left(0, \mu_{n}^{2}\right)$. Corresponding to any solution point $\left(w, \mu^{2}\right) \in \sum_{n}(w \neq 0)$, the symmetry of the string is characterized by the dihedral group $D_{n}$ (the complete symmetry group of a regular $n$-sided polygon for $n \geq 3$; for $n=2$, the complete symmetry group of a rectangle). Moreover, if $W$ is thrice continuously differentiable at $\lambda_{n}$, and if

$$
p^{\prime}\left(\lambda_{n}\right) \neq 0 \text {, }
$$

then corresponding to any "direction,"

$$
\phi_{n}^{\alpha}=\alpha \cos (n s)+(1-\alpha) \sin (n s) \in \operatorname{ker} A\left(\mu_{n}^{2}\right) \quad 0 \leq \alpha \leq 1,
$$

there is a unique, local $D_{n}$-symmetric (pitchfork) branch of bifurcating solutions of the form

$$
\begin{aligned}
& w=x \phi_{n}^{\alpha}+o(x), \\
& \mu^{2}=\mu_{n}^{2}+o(x), \quad \text { as } x \rightarrow 0,
\end{aligned}
$$

where $x \equiv\left\langle\phi_{n}^{\alpha}, w\right\rangle$, and $\langle\cdot, \cdot\rangle$ denotes the usual $L^{2}$ inner product.

Proof. Since the local analysis of $O(2)$-symmetry-breaking bifurcation is well known (cf. [8]), we merely outline the relevant steps here. By (5.14), (5.15), and (5.22), it is easy to show that $\sigma_{n}\left(\tilde{\lambda}\left(\mu^{2}\right)\right)$ is the eigenvalue of the self-adjoint operator $A\left(\mu^{2}\right)$ corresponding to the eigenspace $\operatorname{span}\{\cos (n s), \sin (n s)\}$. In particular, $A\left(\mu^{2}\right)$ is readily shown to be Fredholm of index zero. Well-known group-theoretic arguments justify a standard one-dimensional bifurcational analysis corresponding to the mode $\phi_{n}^{\alpha}$. Hence, both claims of the theorem follow from the equivariant branching 
lemma (e.g., cf. [9]). Indeed, since $\tilde{\lambda}^{\prime}>0$ on $(0, \infty)$ (cf. (2.12)), the first-stated conditions insure an (equivariant) odd-crossing condition, cf. [10]. In particular, we recognize (5.23) as the strict-crossing condition.

By our previous results and Theorem 5.21, we can deduce both necessary and sufficient conditions for bifurcation directly from the graph of (5.16). Specifically, if the curve $y=p(\lambda)$ intersects the line $y=n^{2}-3$ at $\lambda_{n}$, and if $p$ is monotonic on some open neighborhood of $\lambda_{n}$, then bifurcation occurs. Referring to the examples of Fig. 2, we illustrate the associated bifurcation diagrams schematically in Fig. 3.

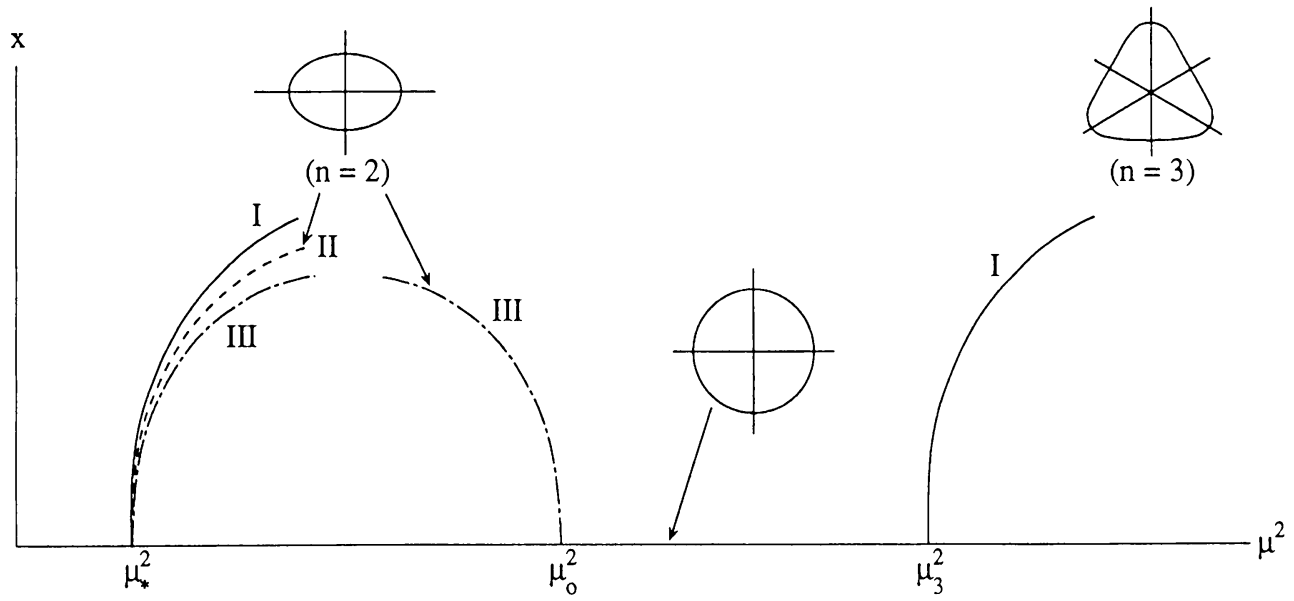

FIG. 3. Bifurcation diagrams corresponding to the three materials depicted in Fig. 2.

If (5.23) holds, then a detailed local analysis shows that the orientation of the pitchfork (subcritical or supercritical) depends upon a maze of terms involving the first through fourth derivatives of $W$ evaluated at $\lambda_{n}$. However, for the special, and perhaps most important, case $n=2$, this expression simplifies considerably, viz., $(5.25 \mathrm{~b})$ is given by

$$
\mu^{2}=\mu_{2}^{2}+\gamma x^{2}+o\left(x^{2}\right), \quad \text { as } x \rightarrow 0,
$$

where

$$
\gamma \equiv\left[48 W^{\prime}\left(\lambda_{2}\right)-162 \lambda_{2}^{2} W^{\prime \prime \prime}\left(\lambda_{2}\right)+189 \lambda_{2}^{3} W^{\prime \prime \prime \prime}\left(\lambda_{2}\right)\right] / 24 \pi \lambda_{2}^{3} p^{\prime}\left(\lambda_{2}\right) .
$$

Of course, $W^{\prime}>0$. However, there are no natural hypotheses for the sign of either $W^{\prime \prime \prime}$ or $W^{\prime \prime \prime \prime}$, which obviates any general conclusions about the sign of $\gamma$.

We complete this section by discussing two important special cases for $n=2$. First suppose that $W^{\prime \prime \prime}\left(\lambda_{2}\right)<0$, with $W^{\prime \prime \prime \prime}\left(\lambda_{2}\right)$ sufficiently small. Then the constitutive law locally resembles that depicted in Fig. $1 \mathrm{~b}$ (with $\lambda_{2} \equiv \lambda_{*}$ ), and the bifurcation diagram is supercritical $(\gamma>0)$, cf. Fig. 3. On the other hand, it is well known that tensile tests (e.g., on rubber) often suggest a constitutive law similar to material III of Fig. 2a, in which case the corresponding graph of $p$ is shown in Fig. 2b. Thus, there are two $(n=2)$ bifurcations in succession, as depicted in Fig. 3. For $\lambda_{0}-\lambda_{*}$ sufficiently small (cf. Fig. $2 \mathrm{a}$ ), we can use standard arguments of singularity 
theory to show that these two branches are connected, forming a continuous "loop" of solutions.

Consider a one-parameter family of (sufficiently smooth) free-energy functions $\widetilde{W}:(0, \infty) \times \mathbb{R} \rightarrow \mathbb{R}$ such that $\lambda \mapsto \widetilde{W}(\lambda, \tau)$ satisfies our original requirements for $W$ (cf. Sec. 2) for all $\tau$ sufficiently small. We further assume that

$$
\widetilde{W}_{\lambda}^{*} / \lambda_{*}=\widetilde{W}_{\lambda \lambda}^{*}>-\widetilde{W}_{\lambda \lambda \tau}^{*}>0, \quad \widetilde{W}_{\tau}^{*}=\widetilde{W}_{\lambda \tau}^{*}=\widetilde{W}_{\lambda \lambda \lambda}^{*}=\widetilde{W}_{\lambda \lambda \lambda \tau}^{*}=0,
$$

and

$$
\widetilde{W}_{\lambda \lambda \lambda \lambda}^{*}>\left|\widetilde{W}_{\lambda \lambda \lambda \lambda \tau}^{*}\right| \geq 0
$$

where $\widetilde{W}_{\lambda}^{*} \equiv \widetilde{W}_{\lambda}\left(\lambda_{*}, 0\right)$, etc. Then the Taylor series of $\widetilde{W}(\lambda, \tau)$ about $\left(\lambda_{*}, 0\right)$ is of the form

$$
\widetilde{W}_{\lambda}(\lambda, \tau)=\widetilde{W}_{\lambda}^{*}+\left(\widetilde{W}_{\lambda}^{*} / \lambda_{*}\right)\left(1-a^{2} \tau\right)\left(\lambda-\lambda_{*}\right)+\left(\widetilde{W}_{\lambda i \lambda \lambda}^{*} / 6\right)\left(1 \pm b^{2}\right)\left(\lambda-\lambda_{*}\right)^{3}+\cdots,
$$

where $0<a^{2}, b^{2}<1$. The constitutive law $T=\widetilde{W}_{\lambda}(\lambda, \tau)$ is illustrated in Fig. 4 for several different small values of the parameter $\tau$. Note that the string is stiff for $\tau<0$ and soft for $\tau \geq 0$.
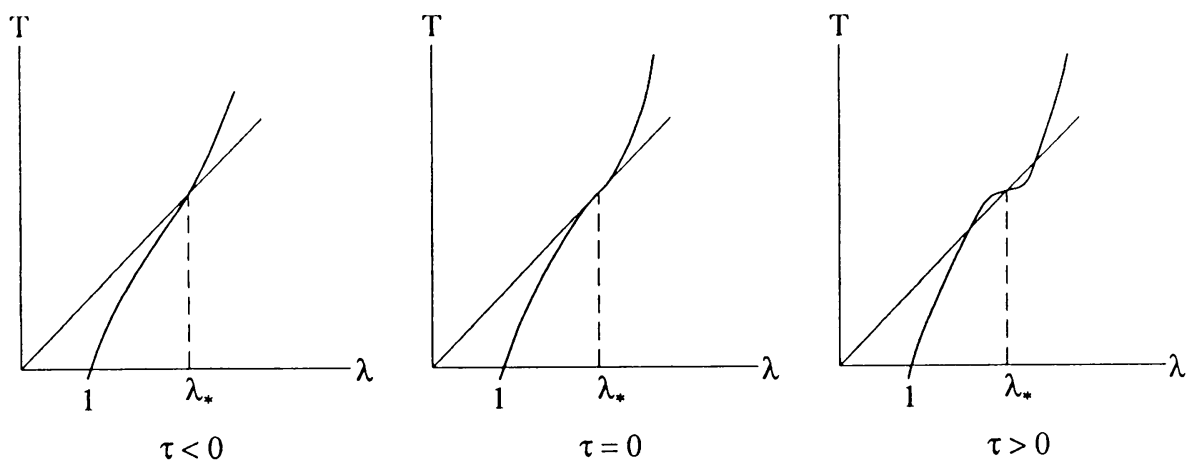

FIG. 4. The constitutive law $T=\widetilde{W}_{\lambda}(\lambda, \tau)$ for three distinguished values of $\tau$.

If we employ $\widetilde{W}$ in place of $W$ in (5.3b) and (5.7), we obtain a two-parameter version of (5.12), denoted by

$$
\tilde{f}\left(w, \mu^{2}, \tau\right)=0,
$$

where $\tilde{f}: \Omega \times(0, \infty) \times \mathbb{R} \rightarrow C_{2 \pi}^{0}$. If we repeat the analysis (5.13)-(5.16) for (5.27), we obtain the two-parameter characteristic equation (for $n=2$ )

$$
\tilde{p}(\lambda, \tau) \equiv \widetilde{W}_{\lambda}(\lambda, \tau) / \lambda \widetilde{W}_{\lambda \lambda}(\lambda, \tau)=1 .
$$

The graph of $\lambda \mapsto \tilde{p}(\lambda, \tau)$ (corresponding to the values of $\tau$ previously considered in Fig. 4) is depicted in Fig. 5. Clearly, (5.28) has no roots, one root, and two roots for $\tau<0, \tau=0$, and $\tau>0$, respectively. Note that $\tau=0$ corresponds to a soft string that does not satisfy the crossing condition (strict or otherwise) of Theorem 5.21 . 


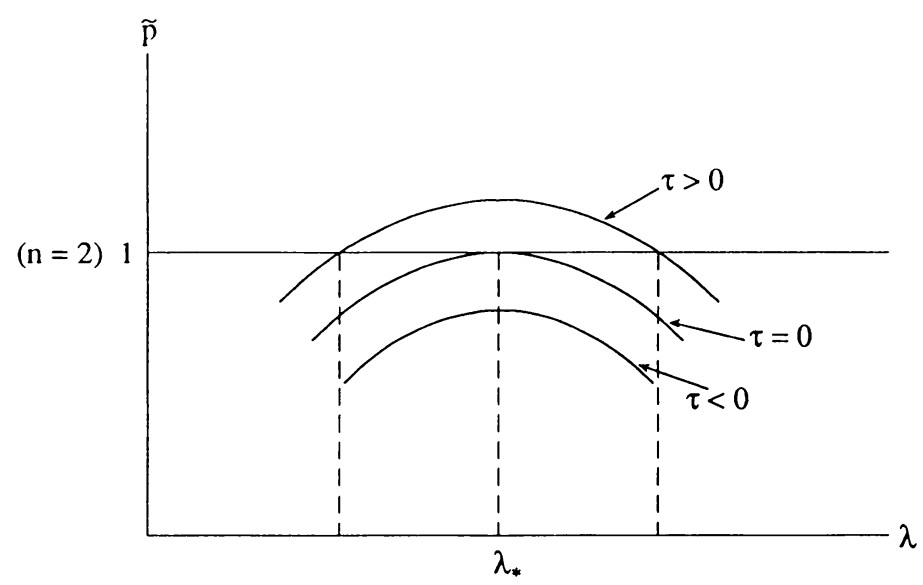

FIG 5. Graph of Eq. (5.28) for three distinguished values of $\tau$.

THEOREM 5.29. For any direction (5.24) $(n=2)$, the local bifurcation diagram of (5.27) near $\left(w, \mu^{2}, \tau\right)=\left(0, \mu_{*}^{2}, 0\right)$ is equivalent to the one generated by the normal form

$$
x^{3}+x\left(\mu^{2}-\mu_{*}^{2}\right)^{2}-x \tau=0,
$$

where $x$ is defined in (5.25b). Hence, the bifurcations are as shown in Fig. 6.

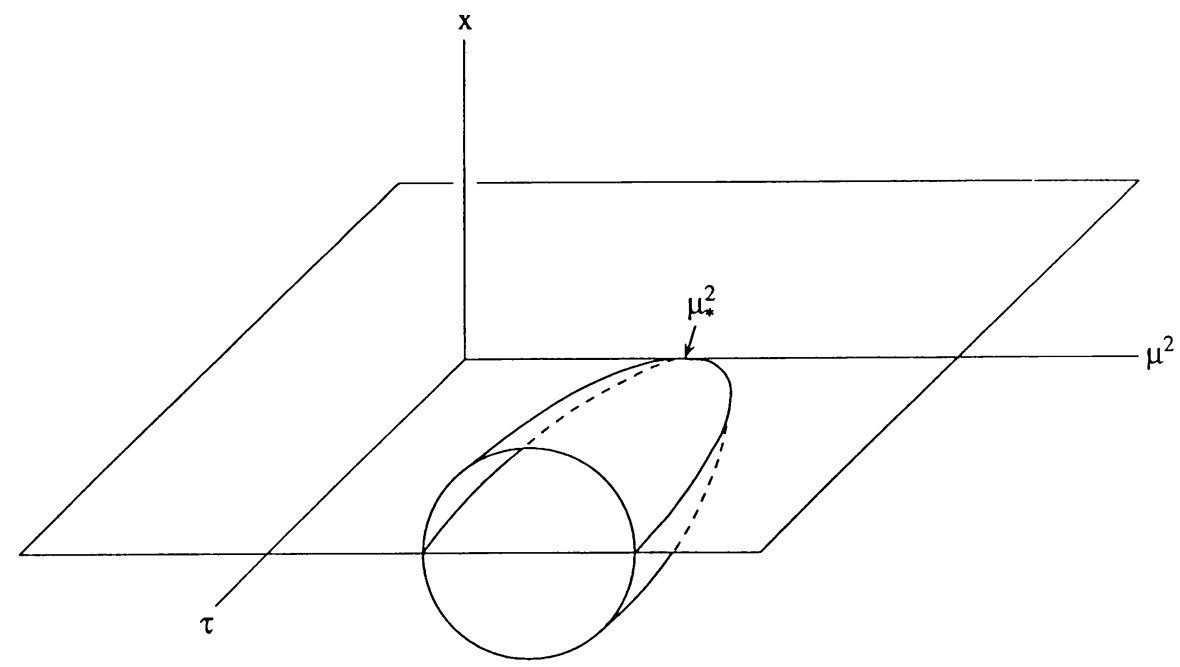

FIG. 6. Bifurcation diagram corresponding to the one-parameter family of constitutive laws $T=\widetilde{W}_{\lambda}(\lambda, \tau)$.

Proof. For any fixed value of $\alpha \in[0,1]$, consider the one-dimensional (LiapunovSchmidt) reduced problem

$$
g\left(x, \mu^{2}, \tau\right) \equiv\left\langle\phi_{2}^{\alpha}, \tilde{f}\left(x \phi_{2}^{\alpha}+\psi\left(x, \mu^{2}, \tau\right), \mu^{2}, \tau\right)\right\rangle=0,
$$


where $\left\langle\phi_{2}^{\alpha}, \psi\left(x, \mu^{2}, \tau\right)\right\rangle=0$ and $\psi\left(0, \mu^{2}, \tau\right)=\psi_{x}\left(0, \mu_{*}^{2}, 0\right)=0$. Since $\left(w, \mu^{2}\right.$, $\tau)=\left(0, \mu_{*}^{2}, 0\right)$ is a singular point on the trivial solution, we know that $g^{*}=g_{x}^{*}=$ 0 , where $g^{*} \equiv g\left(0, \mu_{*}^{2}, 0\right)$, etc., and that all partial derivatives of $g$ with respect to $\mu^{2}$ and $\tau$ evaluated at $x=0$ vanish. Moreover,it is readily shown that $x \mapsto g\left(x, \mu^{2}, \tau\right)$ inherits $Z_{2}$-equivariance (oddness) from the $O(2)$-equivariance of $w \mapsto \tilde{f}\left(w, \mu^{2}, \tau\right)$, cf. [8]. Thus, $g_{x x}^{*}=g_{x x \mu^{2}}^{*}=0$. Finally, several lengthy but straightforward calculations employing conditions (5.26) yield

$$
\begin{gathered}
g_{x \mu^{2}}^{*}=\left\langle\phi_{2}^{\alpha}, D_{w} \tilde{f}_{\mu^{2}}\left(0, \mu_{*}^{2}, 0\right) \phi_{2}^{\alpha}\right\rangle=\tilde{p}_{\lambda}\left(\lambda_{*}, 0\right) \tilde{\lambda}^{\prime}\left(\mu_{*}^{2}\right)=0, \\
g_{x \tau}^{*}=\left\langle\phi_{2}^{\alpha}, D_{u} \tilde{f}_{\tau}\left(0, \mu_{*}^{2}, 0\right) \phi_{2}^{\alpha}\right\rangle=\tilde{p}_{\tau}\left(\lambda_{*}, 0\right) \tilde{\lambda}^{\prime}\left(\mu_{*}^{2}\right)=-\widetilde{W}_{\lambda}^{*} \widetilde{W}_{\lambda \lambda \tau}^{*} / \lambda_{*}\left(\widetilde{W}_{\lambda \lambda}^{*}\right)^{2}>0, \\
g_{x x x}^{*}=\left\langle\phi_{2}^{\alpha}, D_{w}^{3} \tilde{f}\left(0, \mu_{*}^{2}, 0\right) \phi_{2}^{\alpha} \phi_{2}^{\alpha} \phi_{2}^{\alpha}\right\rangle=-\left[48 \widetilde{W}_{\lambda}^{*} / \lambda_{*}^{3}+189 \widetilde{W}_{\lambda \lambda \lambda \lambda}^{*}\right] / 24 \pi<0,
\end{gathered}
$$

and

$$
g_{x \mu^{2} \mu^{2}}^{*}=\left\langle\phi_{2}^{\alpha}, D_{w} \tilde{f}_{\mu^{2} \mu^{2}}\left(0, \mu_{*}^{2}, 0\right) \phi_{2}^{\alpha}\right\rangle=\tilde{p}_{\lambda \lambda}\left(\lambda_{*}, 0\right)\left[\tilde{\lambda}^{\prime}\left(\mu_{*}^{2}\right)\right]^{2}<0 .
$$

Hence, we see that the first few terms of the Taylor series for $g$ agree (in sign) with (5.30). The truncation of the higher-order terms can be justified by well-known methods of singularity theory [11]. In particular, we recognize (5.30) as a universal $Z_{2}$-unfolding of $g\left(x, \mu^{2}, 0\right)$.

Concluding remarks. Referring to Fig. 6, we recall from Sec. 4 that the trivial solution branch is stable to the left of the first bifurcation point. The same analysis implies that the trivial solution is also stable to the right of the second bifurcation point. Of course we expect instability between the two bifurcation points, and the second variation is readily shown to be indefinite there. However, a linear stability analysis of the circular solution in that parameter range is inconclusive; all eigenvalues of the linearized dynamical system are on the imaginary axis. This phenomenon, which is a consequence of the rotational symmetry in the problem, also occurs in the stability analysis of a bead in a rotating bowl of revolution [12], and in that of whirling shafts of circular cross-section [13]. It is interesting to note that the introduction of small dissipation into those simple (finite-dimensional) problems seems to imply instability. Note that we can easily introduce internal damping into our string model (visco-elasticity) without violating conservation of angular momentum, in which case the energy functionals employed in this paper serve as Liapunov functionals. We shall pursue this avenue elsewhere.

Acknowledgments. The work reported herein was supported in part by the Air Force Office of Scientific Research, Grant No. AFOSR-88-0222, and by the U.S. Army Research Office through the Mathematical Sciences Institute of Cornell University, Contract DAAG29-85-C-0018. I am also grateful for the prudent advice of an anonymous reviewer. 


\section{REFERENCES}

[1] T. J. Healey and J. Papadopoulos, Steady axial motions of strings, J. Appl. Mech. (1990), in press

[2] E. J. Routh, stability of a Given State of Motion, Macmillan, London, 1877

[3] D. D. Holm, J. E. Marsden, T. Ratiu, and A. Weinstein, Nonlinear stability of fluid and plasma equilibria, Physics Reports 123, 1-116 (1985)

[4] J. Bailieul and M. Levi, Rotational elastic dynamics, Physica 27b, 43-62 (1987)

[5] V. I. Arnold, V. V. Kozlov, and A. I. Neishtadt, Mathematical Aspects of Classical and Celestial Mechanics, Dynamical Systems III, Encyclopedia of Mathematical Sciences, Ed. V. I. Arnold. Springer-Verlag, New York, 1988

[6] E. Zeidler, Nonlinear Functional Analysis, Vol. III: V'ariational Methods and Optimization, Springer-Verlag, New York, 1985

[7] E. J. Routh, A Treatise on Analytical Statics, Vol. I, Cambridge University Press, Cambridge, 1891

[8] A. Vanderbauwhede, Local Bifurcation and Symmetry, Pitman, Boston, 1982

[9] M. Golubitsky, I. Stewart, and D. G. Schaeffer, Singularities and Groups in Bifurcation Theory, Vol. II, Springer Verlag, New York, 1988

[10] H. Kilehöfer, Multiple eigenvalue bifurcation for Fredholm operators, J. Reine Angew. Math. 358, 104-124 (1985)

[11] M. Golubitsky and D. G. Schaeffer, Singularities and Groups in Bifurcation Theory, Vol. I. Springer Verlag, New York, 1985

[12] H. Lamb, Higher Mechanics, Cambridge University Press, London, 1920

[13] H. Ziegler, Principles of Structural Stability, 2nd ed., Birkhauser Verlag, Basel, 1977 